\title{
CONTROL DE UN RECUPERADOR DE SALES EN UNA PLANTA TERMOSOLAR HÍBRIDA
}

\author{
Pablo Otálora Berenguel ${ }^{1}$, Lidia Roca Sobrino ${ }^{2}$, Javier Bonilla $^{2}$, José Luis Guzmán $^{1}$ \\ ${ }^{1}$ Departamento de Informática, Universidad de Almería, ceiA3, CIESOL, Ctra. Sacramento s/n, \\ 04120 Almería, España. Tel: +34 950 214133; pob452@inlumine.ual.es, joseluis.guzman@ual.es \\ ${ }^{2}$ CIEMAT-Plataforma Solar de Almería, Ctra. de Senés s/n, Tabernas 04200, Almería, Spain; \\ \{lidia.roca,javier.bonilla\}@psa.es
}

\begin{abstract}
Resumen
Este trabajo presenta un sistema de control adaptativo para un recuperador de gases como parte de una planta termosolar híbrida. La variable que se desea controlar en este sistema es la temperatura de salida de las sales, que presenta una dinámica fuertemente no lineal afectada por los flujos másicos de sales y gases. Por ello, el propósito de este artículo ha sido obtener los modelos necesarios para elaborar una estrategia de control con un buen desempeño en todos los puntos de operación, sin necesidad de hacer uso de modelos no lineales, y utilizando esquemas de ajuste de ganancia por tabla e interpolación de parámetros. El algoritmo de control ha sido evaluado por completo en simulación obteniendo resultados satisfactorios.
\end{abstract}

Palabras clave: Termosolar, recuperador de gases, control de procesos, modelado.

\section{Introducción}

Las plantas termosolares son uno de los sistemas de generación de electricidad más prometedores en la actualidad por dos motivos. Por un lado, aprovechan una energía inagotable y totalmente limpia, la energía solar, lo cual supone su mayor ventaja frente a las fuentes de energía tradicionales; mientras que por otro lado, permiten el almacenamiento de la energía producida, lo que las hace destacar entre las energías renovables. En un panorama en que los recursos son cada vez menores, la población se encuentra en constante crecimiento y la contaminación supone un problema grave, las fuentes renovables se han convertido en el centro de atención [6].

El principal problema de la mayoría de fuentes renovables es su incapacidad para ser almacenadas a gran escala. Las baterías electrolíticas no son eficientes más allá de ser usadas en pequeñas instalaciones, y puesto que la generación de energía depende de agentes naturales no controlables, para garantizar un suministro constante es preciso su almacenamiento. La tecnología termosolar se fun- damenta en calentar un fluido térmico (principalmente aceite o sales fundidas) mediante campos solares de concentración. Posteriormente, este fluido entrega su energía a un circuito de agua/vapor para mover una turbina o almacenarla en sales fundidas con el fin de emplearla cuando la demanda lo exija.

Sin embargo, esta energía no siempre será capaz de satisfacer toda la demanda por sí sola. En periodos de alta demanda o baja producción puede resultar insuficiente, lo cual lleva a la creación de plantas de producción híbridas. Una de las configuraciones híbridas más prometedoras son las plantas ISCC (integrated solar combined cycle) que, por lo general, emplean una turbina de gas, un recuperador de gases exhaustos, una turbina de vapor y un campo solar de concentración [3].

En el caso del diseño de planta híbrida del proyecto HYSOL [9], el recuperador de calor aprovecha los gases de salida de la turbina de gas con el fin de calentar las sales fundidas almacenadas, incrementando de esta forma la eficiencia global del sistema y la gestionabilidad de la energía con el fin de mantener la demanda requerida. En el marco del proyecto HYSOL se construyó una planta de demostración en el cluster de innovación termosolar de Manchasol (propiedad de ACS/Cobra), en Castilla La Mancha, España [5] con el fin de evaluar la configuración termosolar híbrida propuesta. A partir del diseño del sistema, se extrajo un modelo teórico y se desarrolló un simulador [5], así como una estrategia de control basada en el modelo no lineal del proceso [4].

El objetivo del presente artículo es elaborar una arquitectura de control basada en el uso de modelos lineales, con el fin de evaluar su desempeño y la eficacia de estrategias de control más simples ante sistemas de dificultad similar. Con este fin, se extraerán una serie de modelos lineales para cada uno de los puntos de operación, y se pondrán a prueba dos estrategias de control basados en estos, la estrategia de ajuste de ganancia por tabla y la de interpolación polinómica de parámetros [2]. Además, se implementará un controlador de parámetros medios que servirá como comparativa de referencia en los distintos puntos de operación. 


\section{Descripción}

El recuperador de gases es un sistema compuesto por una serie de tubos horizontales a través de los cuales se hace circular el fluido térmico que se desea calentar (ver figura 1), siendo sales fundidas en el caso de este trabajo. Estas sales vienen del almacenamiento en frío para dirigirse al caliente, en el que se desea que se mantengan a una temperatura alrededor de $560^{\circ} \mathrm{C}$. Con este fin, es posible modificar el caudal de sales que circula para lograr las especificaciones. Transversalmente a los tubos ascienden los gases de escape de la turbina de biogás, que mediante transferencia de calor por convección incrementan la temperatura del fluido térmico. Este caudal es incontrolable, pues depende de la actuación de la turbina de biogás que se encuentra sujeta a la demanda externa, por lo que es considerado una perturbación [5].

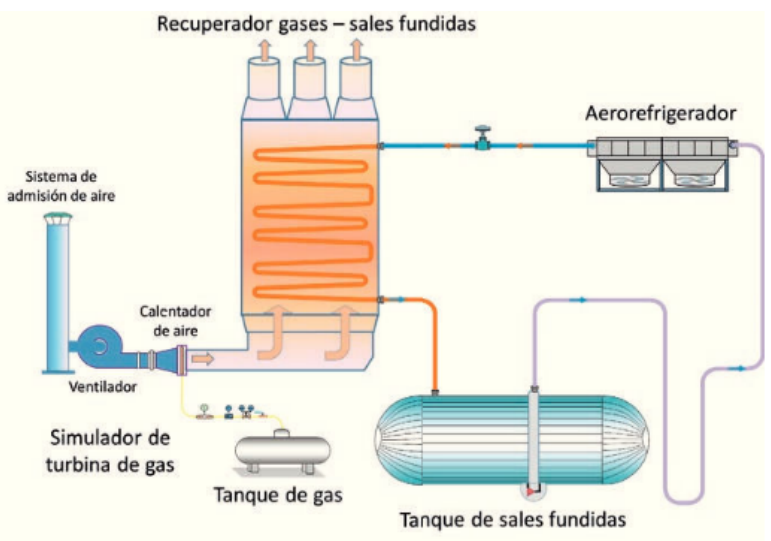

Figura 1: Esquema del proceso con el recuperador de gases [5]

De este modo, es sencillo deducir que para un mayor flujo másico de sales por el recuperador, la temperatura de salida será inferior pues estas se ven expuestas durante menos tiempo a la transferencia de calor. Además, existen ciertos tramos en los extremos de cada tubo del recuperador en los cuales no se produce transferencia alguna, lo cual puede ser interpretado como un tiempo de retardo en el sistema. Al variar el tiempo que el fluido pasa en dichos tramos con el caudal, se observa un retardo variable, un fenómeno que supone una dificultad añadida a la hora de controlar el sistema $[1,8]$.

Se presenta entonces como un sistema con una entrada y una perturbación, y como variable a controlar la temperatura de las sales fundidas. Puesto que la referencia es constante, el tipo de problema será de rechazo a perturbaciones.

\section{Modelado}

El sistema que se desea controlar es altamente no lineal, lo cual supone siempre una dificultad añadida a la hora de ser controlado. Es por ello que no será suficiente con obtener un único modelo lineal del sistema. Si se desea una descripción precisa, es necesario declarar una serie de puntos de operación con el fin de definir un modelo lineal para cada uno de ellos.

Los rangos de flujo másico de sales y de gas son $[1.2,4.8]$ y $[1.4,5.6] \mathrm{kg} / \mathrm{s}$, respectivamente. El primer paso será comprobar que el sistema es controlable para todo el rango de perturbaciones, para lo cual se llevará el flujo másico de gases a sus valores máximo y mínimo y se comprobará si existe algún valor de entrada que consiga llevar a la salida al punto de operación en esas condiciones. Los resultados de este ensayo se muestran en la tabla 1. Se puede deducir que la ganancia del flujo másico de sales es negativa, mientras que la del flujo másico de gas es positiva. También se observa que, mientras que para el flujo másico de gas máximo existe un valor de entrada que acerca la salida al punto de operación, no es así para el valor mínimo, pues en este caso la entrada mínima $(1.2 \mathrm{~kg} / \mathrm{s})$ no consigue subir lo suficiente la temperatura. Por ello, es preciso restringir el rango de perturbaciones en el que se evaluará el sistema, quedando en $[1.85,5.6] \mathrm{kg} / \mathrm{s}$.

Tabla 1: Análisis del rango de perturbación controlable

\begin{tabular}{|c|c|c|}
\hline $\begin{array}{c}\text { Flujo gas } \\
(\mathrm{kg} / \mathrm{s})\end{array}$ & $\begin{array}{c}\text { Flujo sales } \\
(\mathrm{kg} / \mathrm{s})\end{array}$ & $\begin{array}{c}\text { Temperatura } \\
\left({ }^{\mathbf{}} \mathbf{C}\right)\end{array}$ \\
\hline 1.4 & 1.2 & 486 \\
1.4 & 4.8 & 357 \\
5.6 & 1.2 & 650 \\
5.6 & 4.8 & 566 \\
\hline
\end{tabular}

Tras establecer los rangos para cada variable, se fijarán una serie de puntos de operación para cada uno de estos. Con el fin de obtener un número adecuado sin complicar excesivamente el diseño, se ha decidido trabajar con 4 puntos de operación. Puesto que el valor de la temperatura de salida ha de ser constante, y se conoce que cada uno de los flujos másicos posee una dinámica no lineal distinta, se establecerán 4 puntos de operación para cada uno de ellos, equidistantes en magnitud y que cubran todo el rango, siendo estos $[1.2,2.4,3.6 \mathrm{y}$ $4.8] \mathrm{kg} / \mathrm{s}$ para el flujo másico de sales y [1.85, 3.1, 4.35 y 5.6$] \mathrm{kg} / \mathrm{s}$ para el flujo másico de gas. Puesto que sacar un modelo de cada una de las variables para cada combinación de flujos sería excesivo, se ha decidido analizar qué combinaciones acercan la salida al punto de operación, modelando 
Tabla 2: Valor de la salida para combinaciones de flujos

\begin{tabular}{|c|c|c|c|c|}
\cline { 2 - 5 } \multicolumn{1}{c|}{} & \multicolumn{4}{c|}{ Flujo de gas (kg/s) } \\
\hline Flujo de sal (kg/s) & 1.85 & 3.1 & 4.35 & 5.6 \\
\hline 1.2 & 554 & 650 & 650 & 650 \\
\hline 2.4 & 445 & 560 & 650 & 650 \\
\hline 3.6 & 404 & 484 & 570 & 638 \\
\hline 4.8 & 379 & 440 & 504 & 569 \\
\hline
\end{tabular}

únicamente estas. Para ello, se ha comprobado el valor en estado estacionario de cada combinación, cuyos resultados se muestran en la tabla 2 . Se puede deducir que existen cuatro combinaciones que llevan la salida al rango deseado, que son $[1.2$, $1.85],[2.4,3.1],[3.6,4.35]$ y $[4.8,5.6] \mathrm{kg} / \mathrm{s}$ para los flujos másico de sales y gas, respectivamente. Estos serán denominados puntos 1, 2, 3 y 4 en adelante.

A partir de estos puntos, se obtendrán cuatro modelos de cada uno de los flujos, aplicando a cada una de las entradas un escalón de $0.05 \mathrm{~kg} / \mathrm{s}$. Mediante el paquete de MATLAB System Identification Toolbox [7], se logra obtener modelos con alto porcentaje de ajuste para cada caso (superior al 85\%). De este modo, han sido obtenidos los modelos representados en la tabla 3. Todos los modelos obtenidos son sobreamortiguados, de primer o segundo orden, y reflejan claramente la alta no linealidad del sistema.

\section{Diseño}

Tras obtener los modelos pertinentes, se procedió a desarrollar las arquitecturas de control propuestas: el esquema de ajuste de ganancia por tabla y el de interpolación polinomial, así como un controlador de valores medios con el cual realizar comparaciones. Las especificación impuesta es de una constante de tiempo en lazo cerrado 0.2 veces la de bucle abierto.

La primera estrategia desarrollada es la del controlador de valores medios. Para su desarrollo se calculó una planta promedio a partir de los valores de cada uno de los parámetros en cada punto de operación. Esta planta viene definida por las funciones de transferencia de entrada y perturbación mostradas en la ecuaciones (1) y (2).

$$
\begin{gathered}
G(s)=\frac{-105.14775}{2012.97 s+1} e^{-77.1 s} \\
D(s)=\frac{104.35}{(2832.35 s+1)(447.325 s+1)} e^{-87.9 s}
\end{gathered}
$$

A partir de esta planta se sintonizará un controlador PI mediante el método $\lambda$, así como un compensador por adelanto con retardo, representados en las ecuaciones (3) y (4). Este controlador pretende ser una base para realizar las comparaciones pertinentes de los esquemas desarrollados.

$$
\begin{gathered}
C(s)=-0.04\left(1+\frac{1}{2012.97 s}\right) \\
F F(s)=\frac{-0.993(2012.97 s+1)}{(2832.35 s+1)(447.325 s+1)} e^{-10.8 s}
\end{gathered}
$$

A continuación, se sintonizaron los controladores del esquema de ajuste de ganancia por tabla, utilizando los mismo métodos para la sintonía pero partiendo de las funciones de transferencia en cada punto de operación. De este modo, se obtiene un controlador PI y un controlador por adelanto ideal para cada punto, y se realiza la conmutación entre uno y otro en función del punto en que se encuentre el sistema. Los controladores y los compensadores por adelanto sintonizados se muestran en la tabla 4. Las variables que determinan las conmutaciones son el flujo másico de sales para los controladores PI y el de gases para los compensadores por adelanto. El esquema completo del ajuste de ganancia por tabla se muestra en la figura 2.

Tras la sintonización de los bloques pertinentes, se procede a implementar el esquema. Existen dos dificultades a la hora de la implementación. Por un lado, la existencia de acción por adelanto dificulta el tracking, y Antiwindup, por lo que ambas características serán implementadas simultáneamente haciendo uso del modo tracking del bloque PI, e implementando una saturación externa a la señal de control total. El controlador seguirá la señal total saturada, restándole previamente la acción por adelanto, como se muestra en la figura 2 .

Por otro lado, otra dificultad de este esquema es la conmutación de bloques de los compensadores por adelanto. Puesto que cada uno de estos bloques posee una ganancia distinta, en el instante en que la perturbación cambie de punto de operación la acción FeedForward sufrirá un cambio brusco indeseado. En el caso de los controladores PI, este efecto es neutralizado mediante el tracking, regulando el término integral, pero ante la inexistencia de este en los compensadores por adelanto, se ha desarrollado una solución distinta. Esta consiste en sumar la acción de todos los compensadores, pero regulando sus entradas mediante una función. Mientras un compensador no esté activo (la salida no se encuentre en su punto de operación específico), la entrada a este bloque será 
Tabla 3: Funciones de transferencia por punto de operación

\begin{tabular}{|c|c|c|}
\hline Punto & Modelos flujo de sales & Modelos flujo de gas \\
\hline 1 & $\frac{-193.41}{4110.7 s+1} e^{-91.625 s}$ & $\frac{197}{(6384 s+1)(706.89 s+1)} e^{-107.79 s}$ \\
2 & $\frac{-103.07}{1805 s+1} e^{-81.665 s}$ & $\frac{101.76}{(2455.3 s+1)(433.74 s+1)} e^{-94.165 s}$ \\
3 & $\frac{-70.535}{1239.9 s+1} e^{-72.535 s}$ & $\frac{67.487}{(1437.7 s+1)(350.94 s+1)} e^{-81.63 s}$ \\
4 & $\frac{-53.576}{896.28 s+1} e^{-62.575 s}$ & $\frac{51.137}{(1052.4 s+1)(297.73 s+1)} e^{-68 s}$ \\
\hline
\end{tabular}

Tabla 4: Controlador PI y compensador por adelanto por punto de operación

\begin{tabular}{|c|c|c|}
\hline Punto & Controlador PI & FeedForward \\
\hline 1 & $-0.023\left(1+\frac{1}{4110.7 s}\right)$ & $-1.0186 \frac{4110.7 s+1}{(6384 s+1)(706.89 s+1)} e^{-16.165 s}$ \\
2 & $-0.0395\left(1+\frac{1}{1805 s}\right)$ & $-0.987 \frac{1805 s+1}{(2455.3 s+1)(433.74 s+1)} e^{-12.5 s}$ \\
3 & $-0.0548\left(1+\frac{1}{1239.9 s}\right)$ & $-0.957 \frac{1239.9 s+1}{(1437.7 s+1)(350.94 s+1)} e^{-9.095 s}$ \\
4 & $-0.0692\left(1+\frac{1}{896.28 s}\right)$ & $-0.954 \frac{896.28 s+1}{(1052.4 s+1)(297.73 s+1)} e^{-5.425 s}$ \\
\hline
\end{tabular}

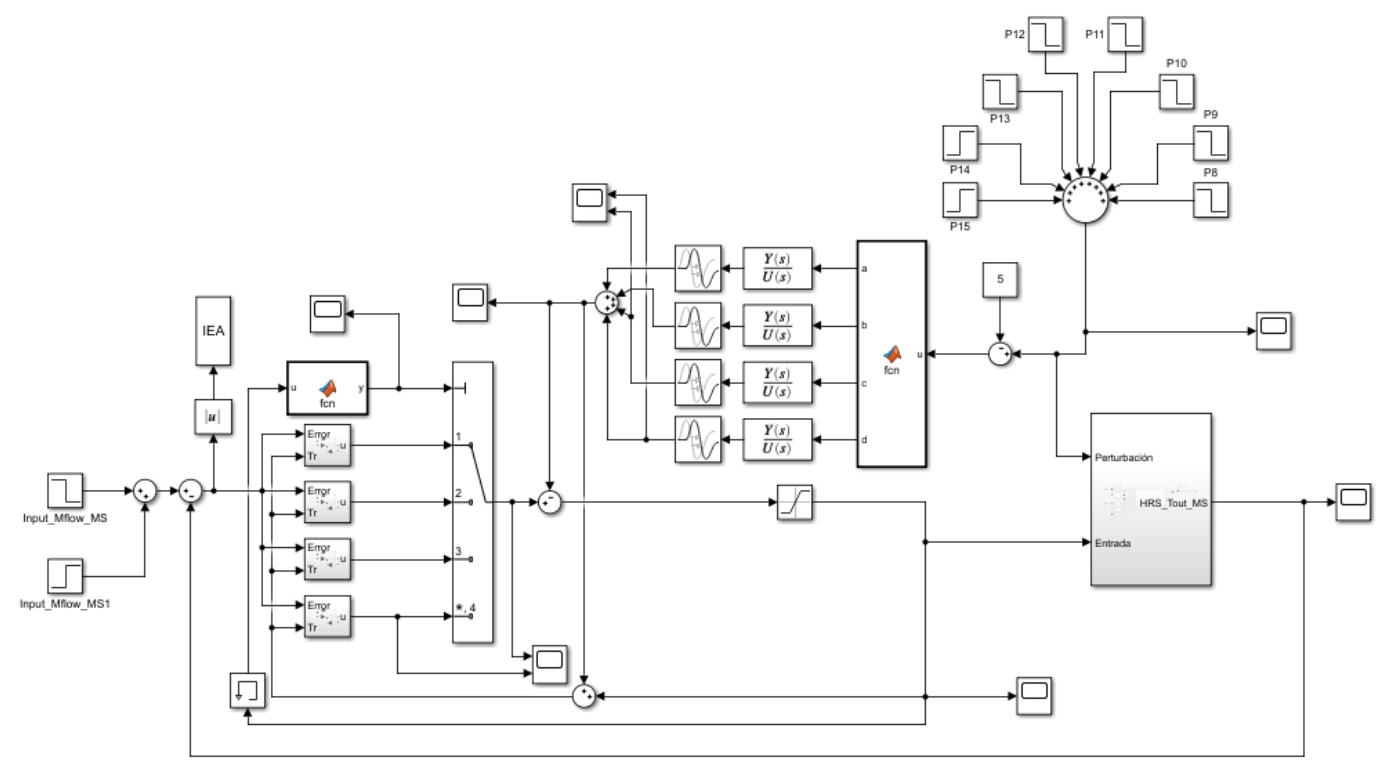

Figura 2: Esquema de control de ajuste de ganancia por tabla

constante e igual a la última entrada que tuvo dicho bloque. Por el contrario, si el bloque sí se encuentra activo, la señal que verá a la entrada será la diferencia entre la señal real total y las señales que entran al resto de bloques inactivos. De este modo, se logra repartir el valor total de la perturbación de modo que el bloque del compensador por adelanto que se encuentre activo sea el único que observe el cambio en la perturbación, y además lo vea únicamente como un incremento de esta, no como su valor absoluto total.

Finalmente, el último esquema implementado es el de interpolación polinómica de parámetros. Se han hallado funciones de tercer orden que relacionan cada uno de los parámetros de las funciones de transferencia con los valores de flujo másico de sales y gas. Estas funciones se representan en la tabla 5. Cabe destacar que todas las funciones son monótonamente crecientes o decrecientes dentro del rango de operación, esto es, no existen puntos de inflexión. En caso contrario, la interpolación sería más inexacta y errática. Los parámetros $K$ hacen referencia a la ganancia estática del sistema, mientras que $\tau$ son las constantes de tiempo $\mathrm{y} t_{r}$ el tiempo de retardo de las respectivas funciones de transferencia del proceso. Por otro lado, el subíndice $G$ indica que son parámetros pertenecientes a la función de transferencia de la entrada, mientras que el subíndice $D$ se aplica a los parámetros de la función de trasferencia de la perturbación. Para su implementación, en el caso del PI fue simple introduciéndolos de manera externa mediante una función, y para los compensadores por adelantó se elaboró un diagrama de bloques que estableciese la función de transferencia según el valor de las salidas de la función. Este esquema se muestra en la figura 3. 
Tabla 5: Polinomio de cada parámetro

\begin{tabular}{|c|c|}
\hline Parámetro & Función \\
\hline$K_{G}$ & $4.073 U^{3}-49.3969 U^{2}+212.0561 U-383.784$ \\
$\tau_{G}$ & $-146.5 U^{3}+1659.3 U^{2}-6418 U+9676.1$ \\
$t r_{G}$ & $-0.1601 U^{3}+1.441 U^{2}-11.8736 U+104.075$ \\
$K_{D}$ & $-3.6731 D^{3}+53.6692 D^{2}-272.9197 D+541.4753$ \\
$\tau_{1 D}$ & $-194 D^{3}+2740 D^{2}-13057 D+22392$ \\
$\tau_{2 D}$ & $-13.7 D^{3}+188.5 D^{2}-894.1 D+1802.7$ \\
$t r_{D}$ & $-0.1865 D^{3}+2.0828 D^{2}-17.7107 D+134.6069$ \\
\hline
\end{tabular}

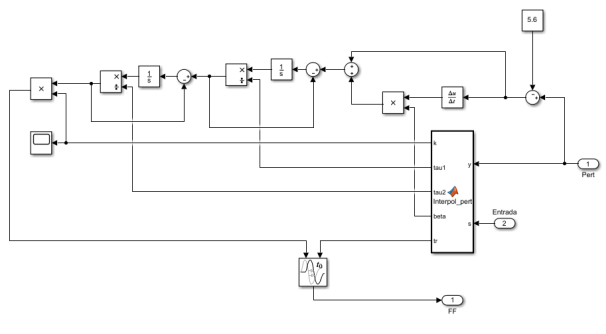

Figura 3: Esquema de compensador por adelanto de parámetros variables

\section{Resultados}

El objetivo de este artículo es comparar la eficacia de los esquemas de control propuestos, para lo cual serán comparados con el control de parámetros medios. Esta comparación se realizará separadamente con acción por adelanto y sin ella, para apreciar la aportación de dicha acción y en qué casos es más valiosa. En el ensayo realizado, el sistema parte de un flujo másico de gas de $5 \mathrm{~kg} / \mathrm{s}$, y se le aplicarán 4 escalones de $-0.5 \mathrm{~kg} / \mathrm{s}$, con el fin de que pase por tres puntos de operación distintos. La referencia es constante de $560^{\circ} \mathrm{C}$. Las figuras 4 y 5 muestran los resultados para el ensayo con y sin compensador por desacopolo, respectivamente.

Como se puede apreciar en las figuras, en el caso de controladores con acción por desacopolo destaca el esquema de ajuste de ganancia por tabla, especialmente en puntos de operación más altos. En los puntos más bajos presentan una dinámica más lenta, la especificación también lo es, no así en el caso del controlador de parámetros medios, que mantiene unas especificaciones constantes intermedias. El esquema de interpolación polinomial con compensador por adelanto es especialmente lento y oscilatorio, lo cual es debido a la constante variación de los parámetros de los compensadores, que dependen del valor de la entrada. Las señales de control son relativamente similares entre sí, a excepción de la del esquema de interpolación polinomial, que es más lenta.

En el caso de los esquemas sin acción compensador por adelanto, son más lentos que aquellos que sí la incorporan como era de esperar, pero presentan una respuesta menos oscilatoria. Los esquemas propuestos son sustancialmente mejores que el controlador de parámetros medios, especialmente en puntos de operación con especificaciones más rápidas. En esta situación las señales de control de las soluciones de ajuste de ganancia e interpolación polinomial son muy próximas entre sí.

Por otro lado, se ha realizado una comparación cuantitativa en función de la integral absoluta del error (IAE) y la integral de la señal de control (ISC). La tabla 6 recoge estos índices. La IAE nos proporciona una medida de la rapidez con la que el sistema rechaza las perturbaciones. Cuanto mayor sea, la salida se ha encontrado más alejada de la referencia a lo largo del ensayo, lo cual es negativo. La ISC representa el esfuerzo de control total empleado en el ensayo, medida de la eficiencia del sistema. El objetivo también es minimizarla. El tiempo de muestreo del ensayo ha sido de 20 segundos, y se tomará la IAE como índice prioritario en la comparativa.

Destaca el esquema de ajuste de ganancia por tabla, que presenta una integral de error muy inferior al resto bajo existencia de acción por adelanto. Sin embargo, el esquema de interpolación polinomial no presenta buenos resultados con esta acción, como se puedo observar en la figura 4, probablemente debido a la rápida variabilidad de esta acción. En cuanto a integral de la señal de control, no varía mucho entre esquemas, siendo inferior para los esquemas con control por adelanto, pues la señal de control disminuye más bruscamente ante cambios en la referencia.

\section{Conclusión}

En este trabajo se ha presentado el control a partir de modelos lineales empíricos de un recuperador de gases. La dificultad fundamental del proceso radicaba en su dinámica altamente no lineal, incluso para el tiempo de retardo. Con el fin de modelarla, se desarrollaron funciones de transferencia estáticas para una serie de puntos de operación definidos. A continuación, se desarrolla- 

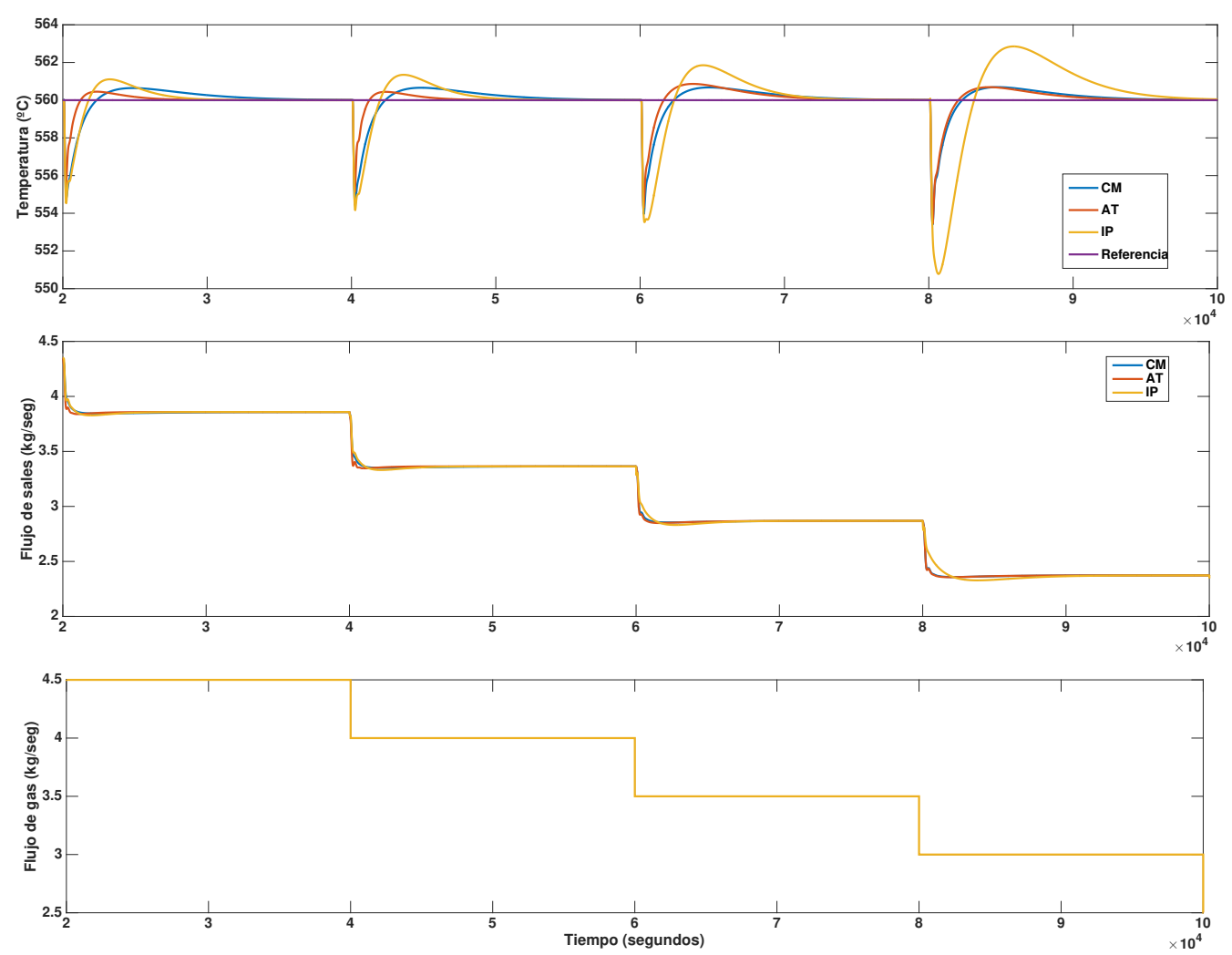

Figura 4: Comparación de controladores con acción por adelanto. Ajuste por Tabla (AT), Interpolación Polinomial (IP), Control Medio (CM).
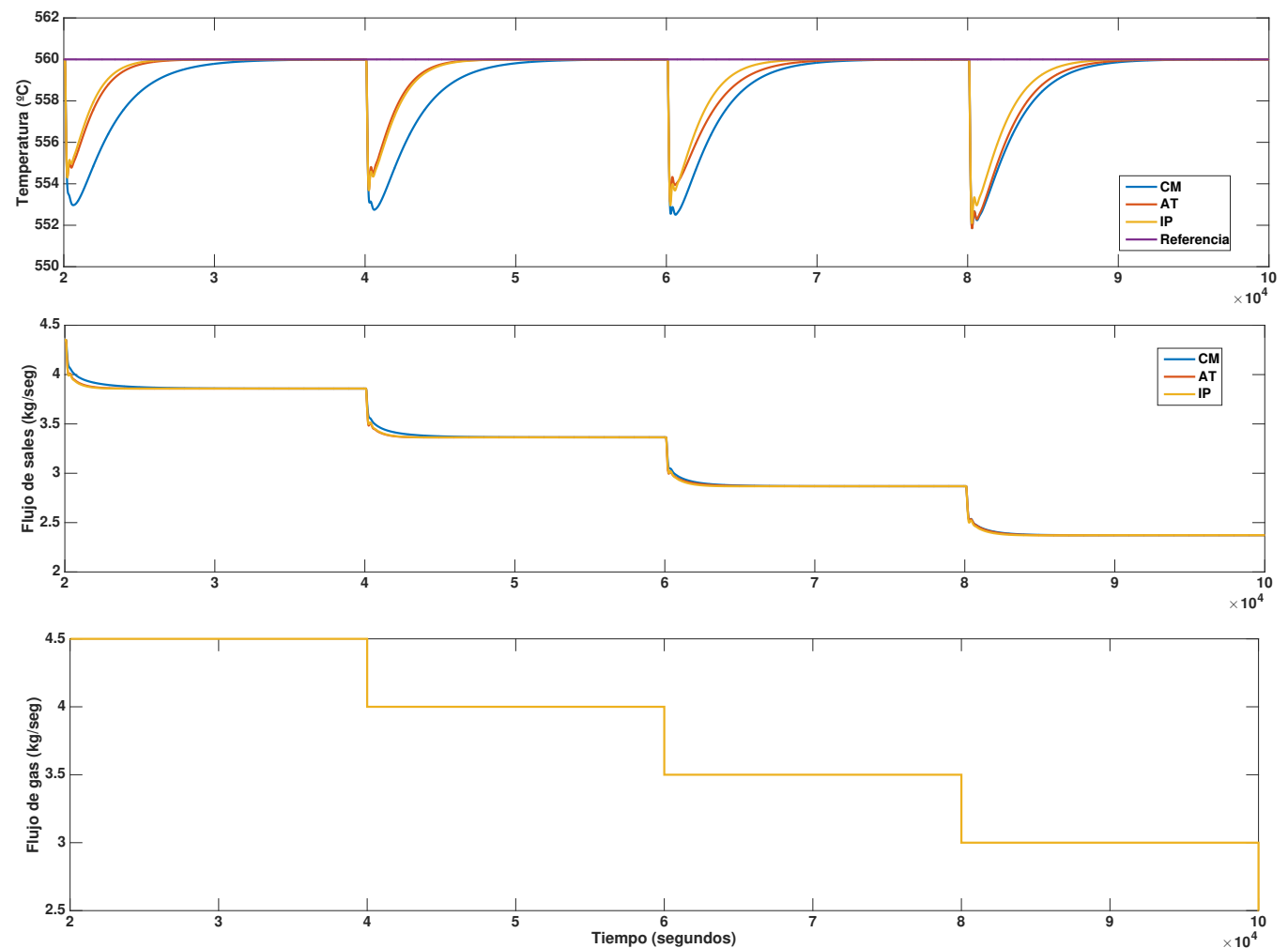

Figura 5: Comparación de controladores sin acción por adelanto. Ajuste por Tabla (AT), Interpolación Polinomial (IP), Control Medio (CM). 
Tabla 6: Índices de las figuras 4 y 5 . Ajuste por Tabla (AT), Interpolación Polinomial (IP), Control Medio (CM), Control por Adelanto (CA)

\begin{tabular}{|l|c|c|}
\hline Estrategia & IAE & ISC \\
\hline AT con CA & 1147.5 & 12474 \\
AT sin CA & 3153 & 12512 \\
IP con CA & 3540.3 & 12472 \\
IP sin CA & 2966.1 & 12510 \\
CM con CA & 1803.4 & 12474 \\
CM sin CA & 4989.1 & 12538 \\
\hline
\end{tabular}

ron los controladores y compensadores por adelanto necesarios para implementar el esquema de control mediante conmutación de ganancias, así como las funciones que permiten la interpolación polinómica de parámetros, logrando un control adaptativo muy satisfactorio. Como líneas futuras se plantea evaluar los esquemas desarrollados en la planta real.

\section{Agradecimientos}

Este trabajo ha sido financiado con el Proyecto DPI2017-85007-R del Ministerio de Economía y Competitividad y Fondos FEDER.

\section{English summary \\ CONTROL OF A HEAT RECOV- ERY SYSTEM IN A HYBRID POWER PLANT}

\begin{abstract}
This paper presents a non-linear control approach for a heat recovery system in a hybrid power plant. The salt temperature is the controlled variable, which presents a highly non-linear dynamics, affected by the salt and gas mass flows. Therefore, the aim of this paper is to obtain the process models to elaborate a control strategy with an adequate performance in the desired operating point, without making use of non-linear models, and employing gain scheduling and parameter interpolation control schemes. The control algorithms have been completely evaluated in simulation obtaining satisfactory results.
\end{abstract}

Keywords: thermosolar, heat recovery system, process control, modelling.

\section{Referencias}

[1] G. Ampuño, L. Roca, J.D. Gil, M. Berenguel, and J.E. Normey-Rico. Apparent delay analysis for a flat-plate solar field model designed for control purposes. Solar Energy, 177(October 2018):241-254, 2019.

[2] K. J. Aström and T. Hägglund. Advanced PID control. The Instrumentation, Systems, and Automation Society, 2006.

[3] Omar Behar, Abdallah Khellaf, Kamal Mohammedi, and Sabrina Ait-Kaci. A review of integrated solar combined cycle system (isccs) with a parabolic trough technology. Renewable and Sustainable Energy Reviews, 39:223-250, 2014.

[4] J. Bonilla and L. Roca. Model validation and control strategy of a heat recovery system integrated in a renewable hybrid power plant demonstrator. Solar Energy, 176(June):698708, 2018.

[5] J. Bonilla, L. Roca, A. De La Calle, and S. Dormido. Modelo Dinámico de un Recuperador de Gases -Sales Fundidas para una Planta Termosolar Híbrida de Energías Renovables. RIAI - Revista Iberoamericana de Automatica e Informatica Industrial, 14(1):70-81, jan 2017.

[6] J. C. Choi, S. D. Kim, and G. Y. Han. Heat transfer characteristics in low-temperature latent heat storage systems using salt-hydrates at heat recovery stage. Solar Energy Materials and Solar Cells, 40(1):71 - 87, 1996.

[7] Contact Mathworks. System Identification Toolbox ${ }^{\text {TM }}$ User's 's Guide R 2014 b. 2014.

[8] J.E. Normey-Rico and E.F. Camacho. Control of Dead-time Processes. 2007.

[9] JF Servert, Eduardo Cerrajero, D López, $\mathrm{S}$ Yagüe, $\mathrm{F}$ Gutierrez, $\mathrm{M}$ Lasheras, and G San Miguel. Base case analysis of a hysol power plant. Energy Procedia, 69:1152-1159, 2015.

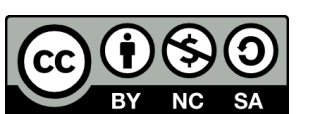

(C) 2019 by the authors. Submitted for possible open access publication under the terms and conditions of the Creative Commons Attribution CC BY-NC-SA 4.0 license (https://creativecommons.org/licenses/by-ncsa/4.0/deed.es). 